\title{
Celecoxib/PLGA Suprime Angiogénesis y Metástasis Pulmonar de un Cáncer Mamario Murino Experimental
}

\author{
Celecoxib/PLGA Suppresses Angiogenesis and Lung \\ Metastasis of Murine Experimental Breast Cancer
}

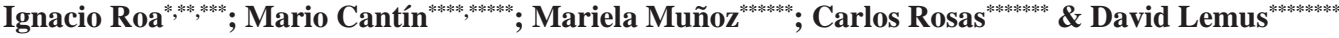

ROA, I.; CANTIN, M.; MUÑOZ, M.; ROSAS, C. \& LEMUS, D. Celecoxib/PLGA suprime angiogénesis y metástasis pulmonar de un cáncer mamario murino experimental. Int. J. Morphol., 34(1):335-341, 2016.

RESUMEN: La angiogénesis y metástasis son eventos esenciales en el proceso de invasión tumoral. Su relación íntima los hace buenos blancos en la terapia antitumoral. El objetivo fue analizar el patrón de metástasis pulmonar y angiogénesis, luego de la aplicación del antiangiogénico Celecoxib microencapsulado en ácido poli(láctico-co-glicólico) en ratones. Se utilizó un modelo de tumor experimental, inducido por células TA3-MTX-R, en 18 ratones, separados en 3 grupos de 6 animales, los cuales fueron tratados con dos presentaciones de Celecoxib en administración intramuscular (Grupo Control; Grupo Cx 1000 ppm y Grupo Cx 1000 ppm+PLGA). Los ratones fueron sacrificados y procesados histológicamente para ser teñidos con H\&E y Tricrómico de Arteta. El estudio reveló que el pulmón muestra una marcada heterogeneidad, y un patrón de metástasis perivascular; además, Celecoxib asociado a ácido poli(lácticoco-glicólico) redujo la invasión tumoral y angiogénesis en el pulmón. Los resultados son similares a descripciones parciales realizadas previamente y son comparables a otras líneas tumorales, siendo celecoxib/ácido poli(láctico-co-glicólico) un candidato potencial en la terapia antitumoral.

PALABRAS CLAVE: Angiogénesis, Metástasis; Pulmón; Cáncer; Celecoxib; PLGA.

\section{INTRODUCCIÓN}

La capacidad de inducir angiogénesis (AG), es una característica crítica de los tumores malignos. La vasculatura del tumor recién formado no sólo proporciona una línea vital de nutrición para el crecimiento tumoral, sino también una ruta de escape clave para la diseminación metastásica (Gavalas et al., 2013). Las actuales terapias antiangiogénicas buscan inhibir o disminuir el proceso de neovascularización y así la invasión.

La Ciclooxigenasa 2 (COX-2) se encuentra presente normalmente en las células y tejidos cerebral y renal, pudiendo ser inducida en estados patológicos tales como inflamación y cáncer. COX-2 es capaz de promover carcinogénesis, proliferación tumoral, AG, la prevención de la apoptosis, y la inmunosupresión (Koki \& Masferrer, 2002), además su sobreexpresión se ha relacionado con el comportamiento desfavorable del tumor y un mal pronóstico en varios tipos de cáncer (Dai et al., 2012; Hill et al., 2012; Shin et al., 2013).

COX-2 se ha convertido así, en un importante blanco en la terapéutica antiangiogénica; debido a la relación existente entre su inhibición y la supresión de la AG y procesos metastásicos (Peng et al., 2013). Su sobreexpresión, en células tumorales afecta la AG debido a la producción de eicosanoides (TAX2; PGI2 y PGE2) (Wang et al., 2013), los cuales estimulan la migración de células endoteliales y AG por el incremento en la expresión del Factor de creci-

\footnotetext{
* Departamento de Ciencias Básicas Biomédicas, Facultad de Ciencias de la Salud, Universidad de Talca, Talca, Chile.

** Programa de Doctorado en Morfología, Facultad de Medicina, Universidad de La Frontera, Temuco, Chile.

**** Becario CONICYT-PCHA/Doctorado Nacional/2015-21150235.

**** CIMA Facultad de Odontología, CEMyQ, Universidad de La Frontera, Temuco, Chile.

****** Centro de Investigación Biomédica, Universidad Autónoma de Chile, Temuco, Chile.

******** Fellow Researcher, Universidad Científica del Sur, Lima, Perú.

******** Departamento de Ciencias Morfológicas, Facultad de Ciencia, Universidad San Sebastián, Santiago, Chile.

********* Laboratorio de Embriología Experimental y Molecular, Programa de Anatomía y Biología del Desarrollo, Instituto de Ciencias Biomédicas, Facultad de Medicina, Universidad de Chile, Santiago, Chile.
} 
miento endotelial vascular (VEGF). Actualmente se busca suprimir dicho evento, para lo cual se han comenzado a emplear inhibidores selectivos de la COX-2, tales como el Celecoxib (Cx), importante agente antinflamatorio (Venkatesan et al., 2011; Rosas et al., 2013, 2014).

Si bien los efectos del $\mathrm{Cx}$ son favorables, su administración oral en altas dosis de manera frecuente, causaría infiltración mononuclear, hiperplasia y degeneración a nivel renal, además de pérdida de algunas funciones hepáticas en animales de experimentación (Kockaya et al., 2010). Por otra parte han sido observados efectos adversos tales como complicaciones sistémicas, gástricas y cardiovasculares (Bomabardier et al., 2000; Silverstein et al., 2000; Cadwell et al., 2006). Con el fin de disminuir estos posibles efectos adversos, al mejorar la biodisponibilidad del Cx, es que este ha sido asociado con micropartículas o nanopartículas poliméricas tales como el ácido poli(láctico-co-glicólico) (PLGA) (Roa et al., 2015).

Puesto que la efectividad de la acción antitumoral del $\mathrm{Cx}$ ha sido relatada previamente y que han sido descritos múltiples efectos adversos en su utilización en altas dosis por largo tiempo, es necesaria la evaluación de nuevas vías de administración tales como las otorgadas gracias a la microencapsulación con PLGA. El objetivo principal del presente estudio es analizar la efectividad del uso de Cx asociado a PLGA, frente a un tumor sólido mamario resistente a metotrexato inducido en modelo murino.

\section{MATERIAL Y MÉTODO}

Línea Tumoral. Se utilizaron células tumorales de adenocarcinoma mamario TA3 en su variedad resistente a metotrexato (TA3-MTX-R), Vinblastina, 5-Fluoruracilo, Cisplatino y CARFENISOP, de crecimiento ascítico, provenientes del Laboratorio de Bioenergética y Cáncer, Programa de Farmacología Molecular y Clínica, Facultad de Medicina, Universidad de Chile. Las células TA3-MTX-R fueron seleccionadas a partir de un tumor ascítico TA3 in vivo, de acuerdo a lo descrito por Rosas et al. (2013), en una concentración final de $2 \times 10^{6}$ células por $\mathrm{mL}$ de la suspensión tumoral.

Fármaco. Celecoxib: Fue utilizado Celecoxib 200 mg (Celebra ${ }^{\circledR}$, Pfizer $^{\odot}$ ), el cual fue diluido en el agua destilada hasta alcanzar la concentración media de 1000 ppm, según lo descrito por Ragel et al. (2007).

Celecoxib/PLGA: Fue encapsulado Celecoxib $200 \mathrm{mg}$ (Celebra ${ }^{\circledR}$, Pfizer $\left.{ }^{\odot}\right)$, en microcápsulas de PLGA de acuerdo a lo descrito por Ayalasomayajula \& Kompella (2005), se obtuvieron micropartículas de entre 0,5 y $10 \mu \mathrm{m}$ de diámetro, en una concentración media de 1000 ppm. La microencapsulación del fármaco fue realizada en el laboratorio de nanoencapsulación, Universidad de Santiago, Chile.

Material Biológico. Fue utilizado un modelo de tumor experimental, inducido por células TA3-MTX-R, en 18 ratones, separados en 3 grupos de 6 animales, los cuales fueron tratados durante 21 días, con dos presentaciones de Cx en administración intramuscular monodosis (Grupo Control: TA3-MTX-R; Grupo Cx 1000 ppm: TA3-MTXR+Cx y Grupo Cx 1000 ppm+PLGA: TA3-MTX-R+Cx/ PLGA). Fueron mantenidos ad libitum, en jaulas conservadas a temperatura ambiente y de acuerdo a las normas impuestas por el Comité de Bioética de la Facultad de Medicina de la Universidad de Chile (CBA \# 0498 FMUCH).

Las muestras de los pulmones fueron fijados en formalina tamponada al $10 \%$ por $48 \mathrm{~h}$, realizándose el procesamiento histológico necesario para obtener cortes de $5 \mu \mathrm{m}$, los que fueron teñidos con H\&E y Tricrómico de Arteta, según lo descrito por Rosas et al. (2013). Se agregaron muestras de pulmón sano de ratón, como control.

Estimación de la invasión tumoral y densidad microvascular. Para la comparación de la invasión tumoral del pulmón se realizó una estimación descriptiva de un campo microscópico de acuerdo a la presencia de células tumorales correspondiente en cada imagen. Para la lectura histológica de las preparaciones se utilizó un microscopio óptico Trinocular PrimoStar (Carl Zeiss, Göttingen, Germany). Las imágenes fueron obtenidas con una cámara digital EOS Rebel XSI (Canon Inc. Japan) acoplada al microscopio y conectada a un computador para las mediciones.

Para la densidad microvascular, se procedió a hacer un conteo de vasos sanguíneos en las muestras histológicas de pulmón con ayuda de un Microscopio trinocular PrimoStar (Carl Zeiss, Göttingen, Germany) con un aumento de 400X, empleando un retículo graduado de $1 \mathrm{~cm}^{2}$ dividido en 10 segmentos.

Análisis estadísticos. La estimación por grupo se realizó observando 30 campos microscópicos con la técnica de doble ciego; registrándose la concordancia utilizando coeficiente kappa para las características cualitativas y coeficiente de Lin para las características cuantitativas, tabulando los datos de acuerdo a lo observado en cada uno de ellos. Se calculó el promedio, error estándar y se aplicó el test $t$ de Student (STATA versión 11.0). 


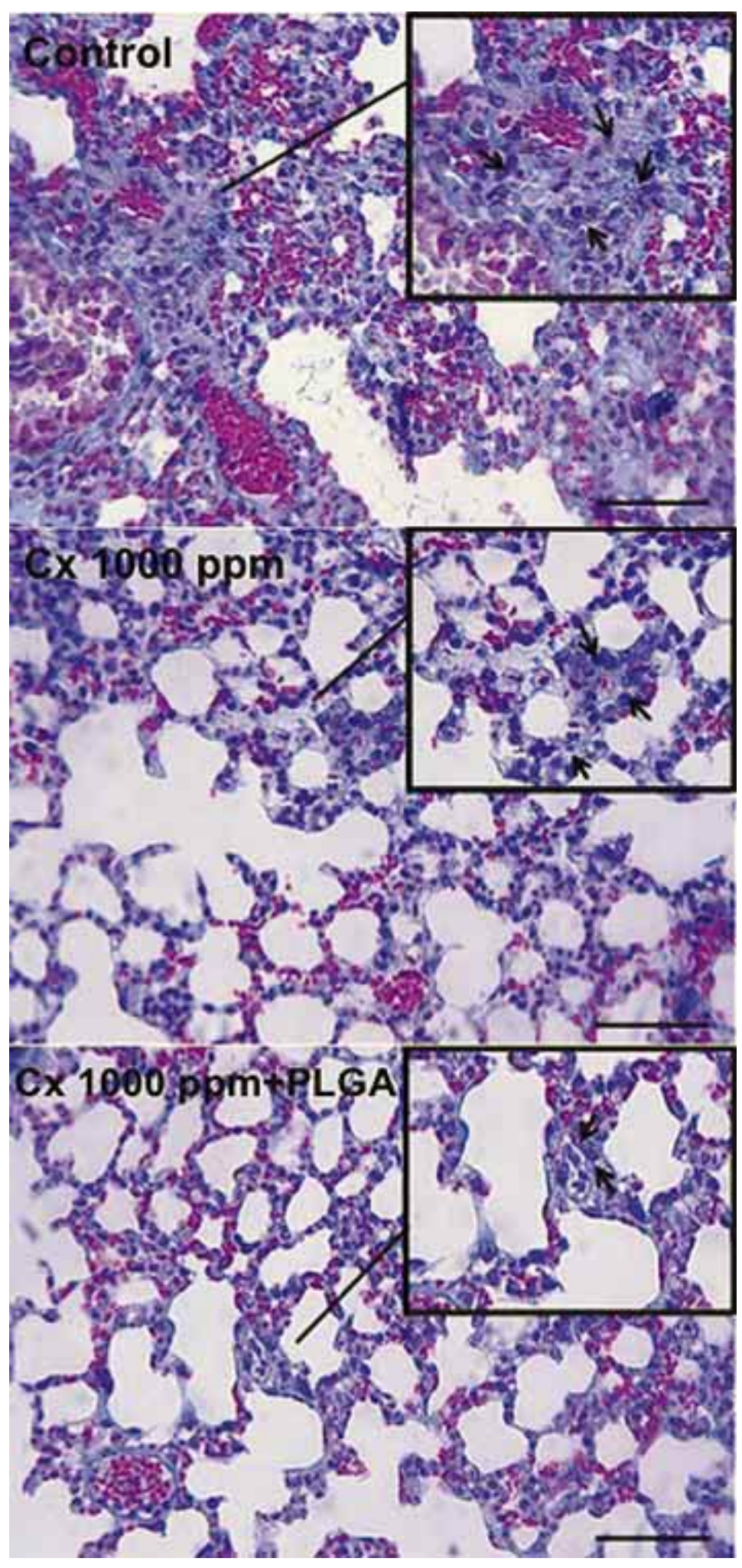

Fig. 1. Aspecto histológico de pulmón de ratón (Mus musculus). Se puede apreciar parénquima pulmonar infiltrado por células tumorales (flechas negras), nótese la asociación de las células tumorales y los vasos sanguíneos (Técnica de Arteta , 400x; barra $100 \mu \mathrm{m}$ ).

\section{RESULTADOS}

Celecoxib microencapsulado disminuye el desarrollo de metástasis pulmonar en ratones inoculados con células TA3-MTX-R.

Histológicamente el grupo Cx 1000 ppm, se caracterizó por presentar una invasión tumoral moderada en pulmón, afectando principalmente el parénquima pulmonar, pudiéndose identificar fácilmente células tumorales. La metástasis fue caracterizada por formar pequeños nódulos celulares bien definidos que deformaban levemente el parénquima pulmonar y se encontraban cercanos a vasos sanguíneos, los cuales se encontraban llenos de eritrocitos, polimorfonucleares y linfocitos. Por su parte el grupo Cx 1000 ppm+PLGA, presentó una invasión tumoral leve a nivel parenquimatoso de características, con acúmulos celulares organizados entorno a vasos sanguíneos. El grupo control, presentó una invasión tumoral abundante. El parénquima pulmonar se vio seriamente afectado por la invasión celular. La población celular era heterogénea al igual que los descritos anteriormente. La metástasis se caracterizó por formar nódulos celulares con forma de "bala de cañón" bien definidos que abarcaban importantes áreas del parénquima perdiendo la forma sacular de los alvéolos y deformando el tejido pulmonar, ubicadas cercanas a abundantes vasos sanguíneos aparentemente formados por la AG tumoral existente en el tejido (Fig. 1).

Cx disminuye densidad microvascular en pulmón.

En los cortes de pulmón de los ratones del Grupo Cx 1000 ppm, se contabilizaron $239,9 \pm 1,807$ vasos $/ \mathrm{cm}^{2}$, a diferencia del Grupo Cx 1000 pppm+PLGA en el que se logró visualizar se obtuvo un promedio de $241,3 \pm 1,758$ vasos $/ \mathrm{cm}^{2}$. Por su parte, el Grupo

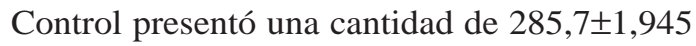
vasos $/ \mathrm{cm}^{2}$ (Fig. 2). Al realizar una comparación de los resultados entre el grupo que fue inoculado con Celecoxib y el que fue inoculado con Celecoxib/PLGA, se logró concluir que no existió una disminución significativa en este último grupo, significancia que sí existió al comparar el grupo control con los otros dos por separado $(\mathrm{P}<0,05)$ (Fig. 2). 


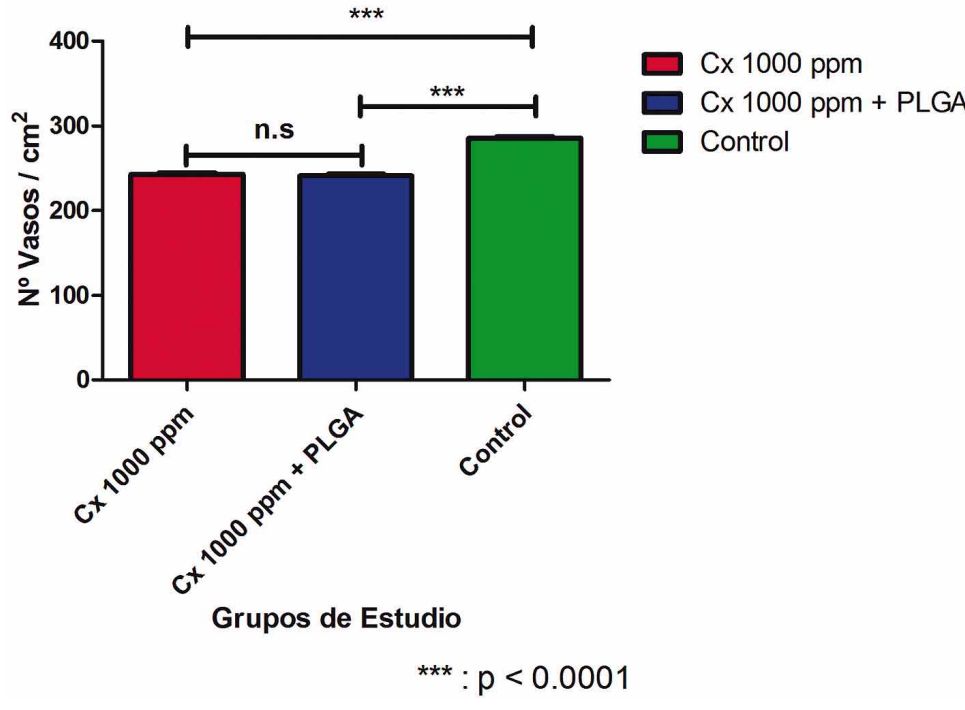

Fig. 2. Densidad microvascular del pulmón por grupo de estudio.

\section{DISCUSIÓN}

Un proceso vital en la progresión del cáncer es la diseminación de las células tumorales, la cual se encuentra íntimamente relacionada con la capacidad del tumor de generar nuevos vasos sanguíneos (Talmadge \& Fidler, 2010). Se ha visto que la COX-2, enzima presente normalmente en los tejidos, se encuentra sobreexpresada en un serie de cánceres (Cianchi et al., 2001; Dempke et al., 2001). Esta sobreexpresión, es observada en casi la mitad de todos los pacientes con cáncer de mama, lo cual marca un signo de mal pronóstico (Ristimäki et al., 2002).

Las estrategias empleadas por las células tumorales son inducidas por numerosos factores proangiogénicos, uno de ellos es el VEGF (Gavalas et $a l$.). Se ha visto que la combinación de $\mathrm{Cx}$ y bloqueadores selectivos de la vía VEGF podría conducir al control de la metástasis en pacientes con cáncer de colon, de mama, $\mathrm{y}$ otros tipos de tumores que sobreexpresan COX-2 (Xu et al., 2014).

En estudios previos de metástasis pulmonar inducida por células TA3-MTX-R, éste presentó signos de invasión por células tumorales, en patrón de "bala de cañón", distribuyéndose a lo largo del pulmón (Rosas et al., 2013, 2014), lo cual es concordante a lo reportado en este estudio, donde la metástasis se caracterizó por formar pequeños nódulos celulares esféricos, bien definidos que deformaron el parénquima pulmonar caracte- rístico. Con respecto a las vías de diseminación tumoral, Labelle \& Hynes (2012) describen la existencia de una vía hematógena que invade a principalmente hígado y pulmones, coincidente a lo encontrado en nuestro estudio.

En el presente estudio, estos focos metastásicos disminuyeron, posterior a la aplicación de $\mathrm{Cx}$, resultados similares a lo encontrado por Rosas et al. (2013, 2014), luego de analizar el mismo modelo experimental de carcinogénesis murina; y a lo visto por Ninomiya et al. (2012), quienes no observaron nódulos metastásicos aparentes, en pulmón, hígado, bazo, riñones, o peritoneo, luego de la administración de Cx. Lo que nos demuestra que $\mathrm{Cx}$ reduce el potencial de crecimiento y metástasis en ratones a través de la inhibición de la COX-2, por efecto antiangiogénico y la inducción de la apoptosis (Ninomiya et al.). Cuando los ratones fueron inoculados con $\mathrm{CX} /$ PLGA, estos presentaron una disminución mayor de los focos metastásicos al compararlos con los grupos control e inoculados solo con $\mathrm{Cx}$, resultados similares a lo relatado por Roa et al., con respecto a metástasis hepática.

En nuestro estudio, Cx redujo la metástasis de células TA3-MTX-R, en pulmón, tanto en su presentación asociada con PLGA, como no microencapsulada. Si bien Cx ha sido previamente reportado su acción frente a metástasis pulmonar (Gendy et al., 2011; Barlow et al., 2012; Zhang et al., 2014) y hueso (Das Roy et al., 2009), y frente a apoptosis y crecimiento tumoral en el mismo modelo (Rosas et al., 2014); no hay información de su efecto frente a metástasis pulmonar cuando es asociado a micropartículas de PLGA.

Con respecto a la AG, COX-2 se ha valorado en neoplasias de múltiples localizaciones, Gallo et al. (2001) demostraron que en tumores de cabeza y cuello existía una correlación significativa entre los niveles de COX-2 y la vascularización tumoral y la densidad de microvasos y VEGF. Asimismo, Uefuji et al. (2000) estudiando adenocarcinomas gástricos hallaron una correlación positiva entre los niveles de COX-2 en las células neoplásicas y la densidad de microvasos, en estos casos la sobreexpresión de COX-2 se relacionaba con niveles elevados de prostaglandinas E2.

Si bien en los resultados encontrados con respecto a la microdensidad vascular, se observa que $\mathrm{Cx}$ y $\mathrm{Cx} /$ PLGA, disminuyen la cantidad de vasos sanguíneos a nivel pulmonar, al ser comparados con el control, no se muestra una diferencia estadísticamente significativa, lo cual podría indicarnos que el modo de presentación del fármaco no fue relevante. Esto podría estar explicado, a que si bien se produce AG en todos los tu- 
mores a medida que crecen, la intensidad de esta puede variar según los tipos de tumor e incluso entre individuos con el mismo tipo de tumor (Eberhard et al., 2000). Se ha descrito que en algunos carcinomas de mama, el aumento de la densidad microvascular no es atribuible, al menos exclusivamente, a la secreción de VEGF por las células tumorales o por los macrófagos, o a la expresión de COX-2 (Leek et al., 2000; Lin et al., 2006), ya que existe activación de la angiogénesis a nivel celular por distintas vías que pueden ser pueden ser inducidas por diversos factores o moléculas que están asociadas para una misma acción (“cross-talking”) (Fulda, 2013).

Cx inhibe la vía de la síntesis de prostaglandinas y hace más lenta la progresión del cáncer, al menos en parte mediante la reducción de las propiedades inmunosupresoras y angiogénicas de PGE2 (Li et al., 2013; Perroud et al., 2013). Además de estas propiedades, se ha visto que PGE2 puede estimular directamente las vías de supervivencia/metástasis de células cancerosas por medio de PI3K, Ras o Wnt/ ß-catenina (Zhou et al., 2005; Wang \& Dubois, 2010). Sui et al. (2014)t por su parte establecen que Cx redujo la expresión de PI3K, P-Akt, COX-2, HIF-12 y VEGF-A, trayendo consigo retraso del crecimiento de un hepatocarcinoma $\mathrm{H} 22$ en modelo murino.

La asociación a PLGA ha sido reportada previamente por Amrite \& Kompella (2008), quienes describieron una reducción significativa de los niveles de VEGF y de PGE2. Esta asociación Cx/PLGA ha sido también utilizada en terapias antiangiogénicas en patologías oculares (Amrite et al., 2006) como en tratamientos antitumorales (Ibrahim et al., 2012). En donde se han visto efectos favorables en la terapia antiangiogénica tales como disminución de la AG e inducción de la apoptosis de las células tumorales (Cui et al., 2010).
$\mathrm{Si}$ bien los resultados al utilizar $\mathrm{Cx}$ son controversiales, este aún logra ser una buena alternativa en la terapia antiangiogénica; solo o en combinación con otras terapias. Como lo visto por Park et al. (2008) en donde ratones tratados con radioterapia y una alta dosis de Cx, retrasó del crecimiento tumoral además de observarse una reducción de las metástasis pulmonares.

La vía de administración podría jugar un rol importante en el proceso de inhibición de la AG y metástasis, por lo cual la exploración de nuevas vías y asociaciones de fármacos favorecerían la investigación en el área de la oncología, en aspectos tales como vascularización. Es así como Cx asociado a PLGA o a otro tipo de polímero son vías factibles de administración de fármacos (Vilos et al., 2012, 2014, 2015) ya que podrían disminuir la frecuencia de administración y la potencial disminución de los efectos adversos, ya que disminuye la magnitud de la cantidad de fármaco liberado en el organismo en el momento de la aplicación. Todo esto agregado a la inocuidad del PLGA al ser administrado en distintos tejidos tales como glándulas salivales o tejido muscular, lo cual lo hace un biomaterial propicio para este tipo de asociaciones (Acuña et al., 2011; Miranda et al., 2013).

\section{CONCLUSIÓN}

En este trabajo, presentamos la acción antiangiogénica y antimetastásica de Cx asociado a micropartículas de PLGA. En donde Cx/PLGA administrado en una única inyección exhibió una mejor efectividad con el Cx no encapsulado en ratones inoculados con células tumorales TA3-MTX-R. Cx/PLGA es un candidato potencial en la terapia antitumoral.

ROA, I.; CANTIN, M.; MUÑOZ, M.; ROSAS, C. \& LEMUS, D. Celecoxib/PLGA suppresses angiogenesis and lung metastasis of murine experimental breast cancer. Int. J. Morphol., 34(1):335-341, 2016.

SUMMARY: Angiogenesis and metastasis are critical events on the tumor invasion process. Their close association is related as a good target in antitumor therapy. The aim was to analyze lung metastasis pattern and angiogenesis following application of microencapsulated Celecoxib with poli(lactic-co-glycolic) acid in mice. An experimental tumor model was assessed, induced by TA3-MTX-R cells, in 18 mice, separated in 3 groups of 6 animals and treated with 2 intramuscular Celecoxib presentations (Group Control; Group Cx 1000 ppm and Group Cx 1000 ppm+PLGA). Mice were sacrificed and histologically processed to stain slides with H\&E and Arteta Trichromic. The study revealed that the lung showed a significant heterogeneity, and a perivascular metastasis pattern; moreover, Celecoxib associated to poli(lactic-co-glycolic) acid reduces tumor invasion and pulmonary angiogenesis. The results are similar to partial previous descriptions and are comparable to other tumor lines, concluding that Celecoxib/poli(lactic-co-glycolic) acid is a potential candidate in antitumor therapy.

KEY WORDS: Angiogenesis; Metastasis, Lung; Cancer; Celecoxib; PLGA. 


\section{REFERENCIAS BIBLIOGRÁFICAS}

Acuña, L.; Suazo Galdames, I.; Zavando, D.; Elgueta, S.; Velásquez, L.; Vilos, C. \& Cantín, M. Morphometric and histopathologic changes in skeletal muscle induced for injectable PLGA microparticles. Int. J. Morphol., 29(2):403-8, 2011.

Amrite, A. C.; Ayalasomayajula, S. P.; Cheruvu, N. P. \& Kompella, U. B. Single periocular injection of celecoxib-PLGA microparticles inhibits diabetes-induced elevations in retinal PGE2, VEGF, and vascular leakage. Invest. Ophthalmol. Vis. Sci., 47(3):1149-60, 2006.

Amrite, A. C. \& Kompella, U. B. Celecoxib inhibits proliferation of retinal pigment epithelial and choroid-retinal endothelial cells by a cyclooxygenase-2-independent mechanism. J. Pharmacol. Exp. Ther, 324(2):749-58, 2008.

Ayalasomayajula, S. P. \& Kompella, U. B. Subconjunctivally administered celecoxib-PLGA microparticles sustain retinal drug levels and alleviate diabetes-induced oxidative stress in a rat model. Eur. $J$. Pharmacol., 511(2-3):191-8, 2005.

Barlow, M.; Edelman, M.; Glick, R. D.; Steinberg, B. M. \& Soffer, S. Z. Celecoxib inhibits invasion and metastasis via a cyclooxygenase 2independent mechanism in an in vitro model of Ewing sarcoma. $J$. Pediatr. Surg., 47(6):1223-7, 2012.

Bombardier, C.; Laine, L.; Reicin, A.; Shapiro, D.; Burgos-Vargas, R.; Davis, B.; Day, R.; Ferraz, M. B.; Hawkey, C. J.; Hochberg, M. C.; Kvien, T. K.; Schnitzer, T. J. \& VIGOR Study Group. Comparison of upper gastrointestinal toxicity of rofecoxib and naproxen in patients with rheumatoid arthritis. VIGOR Study Group. N. Engl. J. Med., 343(21):1520-8, 2000.

Caldwell, B.; Aldington, S.; Weatherall, M.; Shirtcliffe, P. \& Beasley, R. Risk of cardiovascular events and celecoxib: a systematic review and meta-analysis. J. R. Soc. Med., 99(3):132-40, 2006.

Cantín, M.; Miranda, P.; Suazo Galdames, I.; Zavando, D.; Arenas, P.; Velásquez, L. \& Vilos, C. In vivo biocompatibility of the PLGA microparticles in parotid gland. Int. J. Clin. Exp. Pathol., 6(11):24128, 2013.

Cianchi, F.; Cortesini, C.; Bechi, P.; Fantappiè, O.; Messerini, L.; Vannacci, A.; Sardi, I.; Baroni, G.; Boddi, V.; Mazzanti, R. \& Masini, E. Up-regulation of cyclooxygenase 2 gene expression correlates with tumor angiogenesis in human colorectal cancer. Gastroenterology, 121(6):1339-47, 2001.

Cui, F. Y.; Song, X. R.; Li, Z. Y.; Li, S. Z.; Mu, B.; Mao, Y. Q.; Wei, Y. Q. \& Yang, L. The pigment epithelial-derived factor gene loaded in PLGA nanoparticles for therapy of colon carcinoma. Oncol. Rep., 24(3):661-8, 2010.

Dai, Z. J.; Ma, X. B.; Kang, H. F.; Gao, J.; Min, W. L.; Guan, H. T.; Diao, Y.; Lu, W. F. \& Wang, X. J. Antitumor activity of the selective cyclooxygenase-2 inhibitor, celecoxib, on breast cancer in vitro and in vivo. Cancer Cell Int., 12(1):53, 2012.

Das Roy, L.; Pathangey, L. B.; Tinder, T. L.; Schettini, J. L.; Gruber, H. E. \& Mukherjee, P. Breast-cancer-associated metastasis is significantly increased in a model of autoimmune arthritis. Breast Cancer Res., 11(4):R56, 2009.
Dempke, W.; Rie, C.; Grothey, A. \& Schmoll, H. J. Cyclooxygenase-2: a novel target for cancer chemotherapy? J. Cancer Res. Clin. Oncol., 127(7):411-7, 2001.

Eberhard, A.; Kahlert, S.; Goede, V.; Hemmerlein, B.; Plate, K. H. \& Augustin, H. G. Heterogeneity of angiogenesis and blood vessel maturation in human tumors: implications for antiangiogenic tumor therapies. Cancer Res., 60(5):1388-93, 2000.

Fulda, S. How to target apoptosis signaling pathways for the treatment of pediatric cancers. Front. Oncol., 3:22, 2013.

Gallo, O.; Franchi, A.; Magnelli, L.; Sardi, I.; Vannacci, A.; Boddi, V.; Chiarugi, V. \& Masini, E. Cyclooxygenase-2 pathway correlates with VEGF expression in head and neck cancer. Implications for tumor angiogenesis and metastasis. Neoplasia, 3(1):53-61, 2001.

Gavalas, N. G.; Liontos, M.; Trachana, S. P.; Bagratuni, T.; Arapinis, C.; Liacos, C.; Dimopoulos, M. A. \& Bamias, A. Angiogenesis-related pathways in the pathogenesis of ovarian cancer. Int. J. Mol. Sci., 14(8):15885-909, 2013.

Gendy, A. S.; Lipskar, A.; Glick, R. D.; Steinberg, B. M.; Edelman, M. \& Soffer, S. Z. Selective inhibition of cyclooxygenase-2 suppresses metastatic disease without affecting primary tumor growth in a murine model of Ewing sarcoma. J. Pediatr. Surg., 46(1):108-14, 2011.

Hill, R.; Li, Y.; Tran, L. M.; Dry, S.; Calvopina, J. H.; Garcia, A.; Kim, C.; Wang, Y.; Donahue, T. R.; Herschman, H. R. \& Wu, H. Cell intrinsic role of COX-2 in pancreatic cancer development. Mol. Cancer Ther, 11(10):2127-37, 2012.

Ibrahim, M. M.; Abd-Elgawad, A. E.; Soliman, O. A. \& Jablonski, M. M. Nanoparticle-based topical ophthalmic formulations for sustained celecoxib release. J. Pharm. Sci., 102(3):1036-53, 2012.

Kockaya, E. A.; Selmanoglu, G.; Kismet, K. \& Akay, M. T. Pathological and biochemical effects of therapeutic and supratherapeutic doses of celecoxib in Wistar albino male rats. Drug Chem. Toxicol., 33(4):4104, 2010.

Koki, A. T. \& Masferrer, J. L. Celecoxib: a specific COX-2 inhibitor with anticancer properties. Cancer Control, 9(2 Suppl.):28-35, 2002.

Labelle, M. \& Hynes, R. O. The initial hours of metastasis: the importance of cooperative host-tumor cell interactions during hematogenous dissemination. Cancer Discov., 2(12):1091-9, 2012.

Leek, R. D.; Hunt, N. C.; Landers, R. J.; Lewis, C. E.; Royds, J. A. \& Harris, A. L. Macrophage infiltration is associated with VEGF and EGFR expression in breast cancer. J. Pathol., 190(4):430-6, 2000.

Li, W.; Tang, Y. X.; Wan, L.; Cai, J. H. \& Zhang, J. Effects of combining Taxol and cyclooxygenase inhibitors on the angiogenesis and apoptosis in human ovarian cancer xenografts. Oncol. Lett., 5(3):923-8, 2013.

Lin, E. Y.; Li, J. F.; Gnatovskiy, L.; Deng, Y.; Zhu, L.; Grzesik, D. A.; Qian, H.; Xue, X. N. \& Pollard, J. W. Macrophages regulate the angiogenic switch in a mouse model of breast cancer. Cancer Res., 66(23):1123846, 2006

Ninomiya, I.; Nagai, N.; Oyama, K.; Hayashi, H.; Tajima, H.; Kitagawa, H.; Fushida, S.; Fujimura, T. \& Ohta, T. Antitumor and anti-metastatic effects of cyclooxygenase-2 inhibition by celecoxib on human colorectal carcinoma xenografts in nude mouse rectum. Oncol. Rep., 28(3):777-84, 2012. 
Park, W.; Oh, Y. T.; Han, J. H. \& Pyo, H. Antitumor enhancement of celecoxib, a selective Cyclooxygenase-2 inhibitor, in a Lewis lung carcinoma expressing Cyclooxygenase-2. J. Exp. Clin. Cancer Res., 27:66, 2008 .

Peng, L.; Zhou, Y.; Wang, Y.; Mou, H. \& Zhao, Q. Prognostic significance of COX-2 immunohistochemical expression in colorectal cancer: a meta-analysis of the literature. PLoS One, 8(3):e58891, 2013.

Perroud, H. A.; Rico, M. J.; Alasino, C. M.; Queralt, F.; Mainetti, L. E.; Pezzotto, S. M.; Rozados, V. R. \& Scharovsky, O. G. Safety and therapeutic effect of metronomic chemotherapy with cyclophosphamide and celecoxib in advanced breast cancer patients. Future Oncol., 9(3):451-62, 2013.

Ragel, B. T.; Jensen, R. L.; Gillespie, D. L.; Prescott, S. M. \& Couldwell, W. T. Celecoxib inhibits meningioma tumor growth in a mouse xenograft model. Cancer, 109(3):588-97, 2007.

Ristimäki, A.; Sivula, A.; Lundin, J.; Lundin, M.; Salminen, T.; Haglund, C.; Joensuu, H. \& Isola, J. Prognostic significance of elevated cyclooxygenase- 2 expression in breast cancer. Cancer Res., 62(3):632-5, 2002.

Roa, I.; Moraga, J.; Cantín, M.; Rosas, C. \& Lemus, D. Antimetastatic effect of Celecoxib/PLGA in a TA3-MTX-R murine breast adenocarcinoma model. Int. J. Morphol., 33(4):558-65, 2015.

Rosas C., C.; Roa, I.; Sinning O., M.; Fuenzalida B., M. \& Lemus A., D. Celecoxib effect in a multiresistant variant of the TA3 tumor. A histological description. Int. J. Morphol., 31(2):392-8, 2013.

Rosas, C.; Sinning, M.; Ferreira, A.; Fuenzalida, M. \& Lemus, D. Celecoxib decreases growth and angiogenesis and promotes apoptosis in a tumor cell line resistant to chemotherapy. Biol. Res., 47:27, 2014.

Silverstein, F. E.; Faich, G.; Goldstein, J. L.; Simon, L. S.; Pincus, T.; Whelton, A.; Makuch, R.; Eisen, G.; Agrawal, N. M.; Stenson, W. F.; Burr, A. M.; Zhao, W. W.; Kent, J. D.; Lefkowith, J. B.; Verburg, K. M. \& Geis, G. S. Gastrointestinal toxicity with celecoxib vs nonsteroidal anti-inflammatory drugs for osteoarthritis and rheumatoid arthritis: the CLASS study: A randomized controlled trial. Celecoxib Long-term Arthritis Safety Study. JAMA, 284(10):1247-55, 2000

Sui, W.; Zhang, Y.; Wang, Z.; Wang, Z.; Jia, Q.; Wu, L. \& Zhang, W. Antitumor effect of a selective COX-2 inhibitor, celecoxib, may be attributed to angiogenesis inhibition through modulating the PTEN/ PI3K/Akt/HIF-1 pathway in an $\mathrm{H}_{22}$ murine hepatocarcinoma model. Oncol. Rep., 31(5):2252-60, 2014.

Shin, D. M.; Zhang, H.; Saba, N. F.; Chen, A. Y.; Nannapaneni, S.; Amin, A. R.; Müller, S.; Lewis, M.; Sica, G.; Kono, S.; Brandes, J. C.; Grist, W. J.; Moreno-Williams, R.; Beitler, J. J.; Thomas, S. M.; Chen, Z.; Shin, H. J.; Grandis, J. R.; Khuri, F. R. \& Chen, Z. G. Chemoprevention of head and neck cancer by simultaneous blocking of epidermal growth factor receptor and cyclooxygenase-2 signaling pathways: preclinical and clinical studies. Clin. Cancer Res., 19(5):1244-56, 2013.

Talmadge, J. E. \& Fidler, I. J. AACR centennial series: the biology of cancer metastasis: historical perspective. Cancer Res., 70(14):5649-69, 2010.

Uefuji, K.; Ichikura, T. \& Mochizuki, H. Cyclooxygenase-2 expression is related to prostaglandin biosynthesis and angiogenesis in human gastric cancer. Clin. Cancer Res., 6(1):135-8, 2000.
Venkatesan, P.; Puvvada, N.; Dash, R.; Prashanth Kumar, B. N.; Sarkar, D.; Azab, B.; Pathak, A.; Kundu, S. C.; Fisher, P. B. \& Mandal, M. The potential of celecoxib-loaded hydroxyapatite-chitosan nanocomposite for the treatment of colon cancer. Biomaterials, 32(15):3794-806, 2011.

Vilos, C. \& Velasquez, L. A. Therapeutic strategies based on polymeric microparticles. J. Biomed. Biotechnol., 2012:672760, 2012.

Vilos, C.; Constandil, L.; Rodas, P. I.; Cantin, M.; Zepeda, K.; Herrera, N. \& Velasquez, L. A. Evaluation of ceftiofur-PHBV microparticles in rats. Drug Des. Devel. Ther., 8:651-66, 2014.

Vilos, C.; Velasquez, L. A.; Rodas, P. I.; Zepeda, K.; Bong, S. J.; Herrera, N.; Cantín, M.; Simon, F. \& Constandil, L. Preclinical Development and In Vivo Efficacy of Ceftiofur-PLGA Microparticles. PLoS One, 10(4):e0123335, 2015.

Wang, D. \& Dubois, R. N. Eicosanoids and cancer. Nat. Rev. Cancer, 10(3):181-93, 2010.

Wang, X.; Zhang, L.; O'Neill, A.; Bahamon, B.; Alsop, D. C.; Mier, J. W.; Goldberg, S. N.; Signoretti, S.; Atkins, M. B.; Wood, C. G. \& Bhatt, R. S. Cox-2 inhibition enhances the activity of sunitinib in human renal cell carcinoma xenografts. Br. J. Cancer, 108(2):319-26, 2013.

Xu, L.; Stevens, J.; Hilton, M. B.; Seaman, S.; Conrads, T. P.; Veenstra, T. D.; Logsdon, D.; Morris, H.; Swing, D. A.; Patel, N. L.; Kalen, J.; Haines, D. C.; Zudaire, E. \& St. Croix, B. COX-2 inhibition potentiates antiangiogenic cancer therapy and prevents metastasis in preclinical models. Sci. Transl. Med., 6(242):242ra84, 2014.

Zhang, S.; Da, L.; Yang, X.; Feng, D.; Yin, R.; Li, M.; Zhang, Z.; Jiang, F. \& Xu, L. Celecoxib potentially inhibits metastasis of lung cancer promoted by surgery in mice, via suppression of the PGE2-modulated ß-catenin pathway. Toxicol. Lett., 225(2):201-7, 2014.

Zhou, J.; Suzuki, T.; Kovacic, A.; Saito, R.; Miki, Y.; Ishida, T.; Moriya, T.; Simpson, E. R.; Sasano, H. \& Clyne, C. D. Interactions between prostaglandin $\mathrm{E}(2)$, liver receptor homologue-1, and aromatase in breast cancer. Cancer Res., 65(2):657-63, 2005.

Dirección para Correspondencia:
Dr. Ignacio Roa Henríquez
Unidad de Morfología
Departamento de Ciencias Básicas Biomédicas
Facultad de Ciencias de la Salud
Universidad de Talca, Av. Lircay s/n
Talca
CHILE

Email.iroa@utalca.cl

Recibido : 19-11-2015

Aceptado: 18-12-2015 